\title{
Few-baryon interactions from lattice QCD
}

\author{
Takumi Doi for HAL QCD Collaboration
}

Received: date / Accepted: date

\begin{abstract}
We report the recent progress on the determination of three-nucleon forces $(3 \mathrm{NF})$ in lattice QCD. We utilize the Nambu-Bethe-Salpeter (NBS) wave function to define the potential in quantum field theory, and extract two-nucleon forces (2NF) and $3 \mathrm{NF}$ on equal footing. The enormous computational cost for calculating multibaryon correlators on the lattice is drastically reduced by developing a novel contraction algorithm (the unified contraction algorithm). Quantum numbers of the three-nucleon $(3 \mathrm{~N})$ system are chosen to be $\left(I, J^{P}\right)=\left(1 / 2,1 / 2^{+}\right)$(the triton channel), and we extract $3 \mathrm{NF}$ in which three nucleons are aligned linearly with an equal spacing. Lattice QCD simulations are performed using $N_{f}=2$ dynamical clover fermion configurations at the lattice spacing of $a=0.156 \mathrm{fm}$ on a $16^{3} \times 32$ lattice with a large quark mass corresponding to $m_{\pi}=1.13 \mathrm{GeV}$. Repulsive $3 \mathrm{NF}$ is found at short distance.
\end{abstract}

Keywords Lattice QCD · Nuclear Forces · Three-Nucleon Forces

\section{Introduction}

Nuclear (and baryonic) forces are fundamental quantities in nuclear physics, and it is of crucial importance to determine them from the underlying theory, Quantum Chromodynamics (QCD). In particular, the determination of few-baryon forces has a huge impact on not only nuclear physics but also astrophysics.

There are various phenomena where three-nucleon forces $(3 \mathrm{NF})$ are considered to play an important role, e.g., the binding energies of light nuclei [1], the properties of neutron-rich nuclei and the supernova nucleosynthesis 2 and the nuclear equation of state (EoS) as well as the saturation point of nuclear matter 3. Deuteron-proton elastic scattering experiments have also shown a clear indication of $3 \mathrm{NF}$ [4,5]. Recent observational data on the maximum mass of neutron stars [6] triggered renewed interest in the nuclear EoS at high density, and universal short-range repulsion for three baryons (nucleons and hyperons) may be needed in neutron stars with hyperon core [7,8].

T. Doi

Theoretical Research Division, Nishina Center, RIKEN, Wako 351-0198, Japan

E-mail: doi@ribf.riken.jp 
Despite of its phenomenological importance, microscopic understanding of $3 \mathrm{NF}$ is still limited. Pioneered by Fujita and Miyazawa [9], the long range part of 3NF has been commonly modeled by the two-pion exchange $(2 \pi \mathrm{E})$, particularly with the $\Delta$-resonance. This $2 \pi \mathrm{E}-3 \mathrm{NF}$ is known to have an attractive nature at long distance, and is considered to play a dominant role to resolve the problem that light nuclei are underbound with $2 \mathrm{NF}$ only. Unfortunately, the $2 \pi \mathrm{E}-3 \mathrm{NF}$ component alone cannot represent the whole properties of 3NF: For instance, it would provide too strong attraction, and thus incurs the overbound problem for light nuclei. In order to handle this issue, an additional repulsive component of $3 \mathrm{NF}$ at short distance is often introduced. In Tucson-Melbourne model [10, a phenomenological cut-off parameter, $\Lambda \simeq 0.7 \mathrm{GeV}$, is introduced in the (monopole) form factor of $\pi N N$ vertex 11. Compared to $\Lambda \simeq 1.3 \mathrm{GeV}$ obtained from $N N$ scattering data [12, one observes that short range repulsion is implicitly added in $3 \mathrm{NF}$. In Urbana/Illinois models, on the other hand, a repulsive $3 \mathrm{NF}$ component at short distance is explicitly introduced in a purely phenomenological way [13. Recently, an approach based on the chiral effective field theory $(\chi \mathrm{EFT})$ is also developing [14, 15, and found to be useful to classify and parametrize the two-, three- and more-nucleon forces. The $\chi \mathrm{EFT}-3 \mathrm{NF}$ contains not only pion exchange terms but also short range terms. The LECs $\left(c_{D}, c_{E}\right)$ corresponding to the latter are known to be sensitive to the cut-off parameter in $\chi \mathrm{EFT}$ [16,17. We note that repulsive short-range 3NF component is phenomenologically required to explain the properties of high density matter $[3,7,8]$ and its detailed information is one of the central question of interest.

Since $3 \mathrm{NF}$ is originated by the fact that a nucleon is not a fundamental particle, it is most desirable to determine $3 \mathrm{NF}$ directly from QCD, in particular at short distance where the dynamics of fundamental degrees of freedom (DoF), quarks and gluons, becomes essential. In this proceeding, we report our progress on first-principles calculations of $3 \mathrm{NF}$ using lattice QCD simulations [18,19. Note that while there are lattice QCD works for three- (or more) baryon systems [20,21,22, 23, they focus on the energies of the multi-baryon systems, and extracting $3 \mathrm{NF}$ is currently beyond their scope.

As for the calculation of two-nucleon forces (2NF) from lattice QCD, an approach based on the NBS wave function has been proposed [24,25, 26], so that the potential is faithful to the phase shift by construction. Resultant (parity-even) $2 \mathrm{NF}$ are found to have desirable features such as attractive wells at long and medium distances and central repulsive cores at short distance. The method has been applied to general hadronic interactions $27,28,29,30,31,32,33,34,35$. The method itself has been also generalized to "time-dependent" HAL QCD method, so that the energy-independent (non-local) potential can be extracted without ground state saturation 36. See Refs. 26, 37, 38 for recent reviews. In this report, we extend the method to three-nucleon $(3 \mathrm{~N})$ systems, and perform the lattice QCD simulations for 3NF in the triton channel, $\left(I, J^{P}\right)=\left(1 / 2,1 / 2^{+}\right)$[18, 19]. In particular, we update the results in Ref. 19] by employing the time-dependent HAL QCD method [36] to suppress the systematic error associated with excited states. We also utilize a novel contraction algorithm (unified contraction algorithm), which reduces the enormous computational cost for $3 \mathrm{~N}$ correlators by a factor of 192 [39.

\section{Formalism}

We first briefly explain the framework for the calculation of $2 \mathrm{~N}$ potentials $26,37,38$. The central quantity in the HAL QCD method is the equal-time Nambu-Bethe-Salpeter 
(NBS) wave function, $\phi_{2 N}(\mathbf{r}) \equiv\left\langle 0|N(\mathbf{r}) N(\mathbf{0})| E_{2 N}\right\rangle$, where $\left|E_{2 N}\right\rangle$ denotes the state of the $2 \mathrm{~N}$ system at the energy of $E_{2 N}$ in the center-of-mass frame, $N$ the nucleon operator where spinor/flavor indices are implicit. For simplicity, we consider the elastic region hereafter, while the method can be extended above inelastic threshold 31, 40. The important property of the wave function is that it has a desirable asymptotic behavior,

$$
\phi_{2 N} \propto \frac{\sin \left(k r-l \pi / 2+\delta_{l}^{k}\right)}{k r}, \quad r \equiv|\mathbf{r}| \rightarrow \infty,
$$

where $E_{2 N}=k^{2} /(2 \mu)$ with the reduced mass $\mu=m_{N} / 2, l$ being the orbital angular momentum. Exploiting this feature, we define the (non-local) $2 \mathrm{~N}$ potential, $U_{2 N}\left(\mathbf{r}, \mathbf{r}^{\prime}\right)$, through the following Schrödinger equation,

$$
-\frac{\nabla^{2}}{2 \mu} \phi_{2 N}(\mathbf{r})+\int d \mathbf{r}^{\prime} U_{2 N}\left(\mathbf{r}, \mathbf{r}^{\prime}\right) \phi_{2 N}\left(\mathbf{r}^{\prime}\right)=E_{2 N} \phi_{2 N}(\mathbf{r}) .
$$

It is evident that, although $U_{2 N}$ itself is not an observable, $U_{2 N}$ is always faithful to the phase shift by construction. Another important property is that, while $U_{2 N}$ could be energy-dependent in general, it was proven that one can construct $U_{2 N}$ so that it becomes energy-independent [25, 26, 38, 40].

In lattice QCD, the NBS wave function of the ground state, $\phi_{2 N}^{0}$, can be extracted from the four-point correlator as

$$
\begin{aligned}
G_{2 N}\left(\mathbf{r}, t-t_{0}\right) & \equiv \frac{1}{L^{3}} \sum_{\mathbf{R}}\left\langle 0\left|(N(\mathbf{R}+\mathbf{r}) N(\mathbf{R}))(t) \overline{\left(N^{\prime} N^{\prime}\right)}\left(t_{0}\right)\right| 0\right\rangle \\
& \underset{t \gg t_{0}}{\longrightarrow} A_{2 N}^{0} \phi_{2 N}^{0} e^{-E_{2 N}^{0}\left(t-t_{0}\right)}, \quad A_{2 N}^{0}=\left\langle E_{2 N}^{0}\left|\overline{\left(N^{\prime} N^{\prime}\right)}\right| 0\right\rangle, \\
\phi_{2 N}^{0}(\mathbf{r}) & \equiv\left\langle 0|N(\mathbf{r}) N(\mathbf{0})| E_{2 N}^{0}\right\rangle,
\end{aligned}
$$

where $E_{2 N}^{0}$ denotes the energy of the ground state, $N\left(N^{\prime}\right)$ the nucleon operator in the sink (source). In the practical lattice calculation, however, it is notoriously difficult to achieve the ground state saturation [36. This is because (i) the energy splitting between the ground state and excited states are getting smaller for larger lattice volume and (ii) signal to noise ratio $(\mathrm{S} / \mathrm{N})$ in lattice Monte Carlo simulation is ruined exponentially with increasing $t$. $\mathrm{S} / \mathrm{N}$ also becomes exponentially worse for larger mass number in the system, and/or for lighter pion mass on the lattice.

In order to overcome this problem, it is recently proposed [36] to consider the time-dependent Schrödinger equation,

$$
-\frac{\nabla^{2}}{2 \mu} \psi_{2 N}(\mathbf{r}, t)+\int d \mathbf{r}^{\prime} U_{2 N}\left(\mathbf{r}, \mathbf{r}^{\prime}\right) \psi_{2 N}\left(\mathbf{r}^{\prime}, t\right)=-\frac{\partial}{\partial t} \psi_{2 N}(\mathbf{r}, t)
$$

where $\psi_{2 N}$ is imaginary-time NBS wave function defined by

$$
\psi_{2 N}(\mathbf{r}, t) \equiv G_{2 N}(\mathbf{r}, t) / e^{-2 m_{N} t}
$$

What is noteworthy is that, thanks to the energy-independence of $U_{2 N}$, Eq. (66) holds not only for the ground state but also for excited states simultaneously. Therefore, the ground state saturation is not required, which is a significant advantage of the potential approach to multi-baryon systems in lattice QCD.

In the practical calculation, we perform the derivative expansion for the non-locality of the potential, $U_{2 N}\left(\mathbf{r}, \mathbf{r}^{\prime}\right)=\left[V_{C}(r)+V_{T}(r) S_{12}+V_{L S}(r) \mathbf{L} \cdot \mathbf{S}+\mathcal{O}\left(\nabla^{2}\right)\right] \delta\left(\mathbf{r}-\mathbf{r}^{\prime}\right)$, 
where $V_{C}, V_{T}$ and $V_{L S}$ are the central, tensor and spin-orbit potentials, respectively. In Ref. [33, the validity of this expansion is examined, and it is shown that the leading terms, $V_{C}$ and $V_{T}$, dominate the potential at low energies.

We now extend the method to the $3 \mathrm{~N}$ system. We consider the imaginary-time NBS wave function of the $3 \mathrm{~N}, \psi_{3 N}(\mathbf{r}, \boldsymbol{\rho}, t)$, defined by the six-point correlator as

$$
\begin{aligned}
G_{3 N}\left(\mathbf{r}, \boldsymbol{\rho}, t-t_{0}\right) & \equiv \frac{1}{L^{3}} \sum_{\mathbf{R}}\left\langle 0\left|\left(N\left(\mathbf{x}_{1}\right) N\left(\mathbf{x}_{2}\right) N\left(\mathbf{x}_{3}\right)\right)(t) \overline{\left(N^{\prime} N^{\prime} N^{\prime}\right)}\left(t_{0}\right)\right| 0\right\rangle, \\
\psi_{3 N}\left(\mathbf{r}, \boldsymbol{\rho}, t-t_{0}\right) & \equiv G_{3 N}\left(\mathbf{r}, \boldsymbol{\rho}, t-t_{0}\right) / e^{-3 m_{N}\left(t-t_{0}\right)}
\end{aligned}
$$

where $\mathbf{R} \equiv\left(\mathbf{x}_{1}+\mathbf{x}_{2}+\mathbf{x}_{3}\right) / 3, \mathbf{r} \equiv \mathbf{x}_{1}-\mathbf{x}_{2}, \boldsymbol{\rho} \equiv \mathbf{x}_{3}-\left(\mathbf{x}_{1}+\mathbf{x}_{2}\right) / 2$ are the Jacobi coordinates. With the derivative expansion of the potentials, the NBS wave function can be converted to the potentials through the following Schrödinger equation,

$\left[-\frac{1}{2 \mu_{r}} \nabla_{r}^{2}-\frac{1}{2 \mu_{\rho}} \nabla_{\rho}^{2}+\sum_{i<j} V_{2 N}\left(\mathbf{r}_{i j}\right)+V_{3 N F}(\mathbf{r}, \boldsymbol{\rho})\right] \psi_{3 N}(\mathbf{r}, \boldsymbol{\rho}, t)=-\frac{\partial}{\partial t} \psi_{3 N}(\mathbf{r}, \boldsymbol{\rho}, t)$,

where $V_{2 N}\left(\mathbf{r}_{i j}\right)$ with $\mathbf{r}_{i j} \equiv \mathbf{x}_{i}-\mathbf{x}_{j}$ denotes the $2 \mathrm{NF}$ between $(i, j)$-pair, $V_{3 N F}(\mathbf{r}, \boldsymbol{\rho})$ the $3 \mathrm{NF}, \mu_{r}=m_{N} / 2, \mu_{\rho}=2 m_{N} / 3$ the reduced masses. If we calculate $\psi_{3 N}(\mathbf{r}, \boldsymbol{\rho}, t)$, and if all $V_{2 N}\left(\mathbf{r}_{i j}\right)$ are obtained by (separate) lattice calculations for genuine $2 \mathrm{~N}$ systems, we can extract $V_{3 N F}(\mathbf{r}, \boldsymbol{\rho})$ through Eq. (10).

One of the greatest challenges in the study of multi-baryon systems on the lattice is that the computational cost of the correlators is exceptionally enormous. Actually, for larger mass number in the system, the cost for color/spinor contractions grows exponentially, and the cost for Wick contractions grows factorially: The total cost is the multiplication of both costs. In order to meet this challenge, we recently develop a novel algorithm in which color/spinor contractions and Wick contractions are considered simultaneously in a unified contraction index list [39. In this "unified contraction algorithm," redundancies in the original contractions are eliminated, and a significant reduction in the computational cost is achieved, e.g., by a factor of 192 for ${ }^{3} \mathrm{H}$ and ${ }^{3} \mathrm{He}$ correlators, and a factor of 20736 for the ${ }^{4} \mathrm{He}$ correlator. We note that, since a potential is independent of the choice of a nucleon operator at the source, we here employ the non-relativistic limit operator at the source to maximize the gain by the unified contraction algorithm. For the nucleon operator at the sink, which defines the NBS wave function (and correspondingly, the "scheme" of the potential 26, 37, 38), we employ the standard nucleon operator, $N_{s t d} \equiv \epsilon_{a b c}\left(q_{a}^{T} C \gamma_{5} q_{b}\right) q_{c}$, for both of $2 \mathrm{NF}$ and $3 \mathrm{NF}$, so that they are determined on the same footing.

In our first exploratory study of $3 \mathrm{NF}$, we restrict the geometry of the $3 \mathrm{~N}$. More specifically, we consider the "linear setup" with $\boldsymbol{\rho}=\mathbf{0}$, with which $3 \mathrm{~N}$ are aligned linearly with equal spacings of $r_{2} \equiv|\mathbf{r}| / 2$. In this setup, the third nucleon is attached to $(1,2)$-nucleon pair with only $\mathrm{S}$-wave. Considering the total $3 \mathrm{~N}$ quantum numbers of $\left(I, J^{P}\right)=\left(1 / 2,1 / 2^{+}\right)$, the triton channel, the wave function can be completely spanned by only three bases, which can be labeled by the quantum numbers of $(1,2)$-pair as ${ }^{1} S_{0},{ }^{3} S_{1},{ }^{3} D_{1}$. Therefore, the Schrödinger equation leads to the $3 \times 3$ coupled channel equations with the bases of $\psi_{1} S_{0}, \psi_{3} S_{1}, \psi_{3} D_{1}$. The reduction of the dimension of bases is expected to improve the $\mathrm{S} / \mathrm{N}$ as well. It is worth mentioning that considering the linear setup is not an approximation: Among various geometric components of the wave function in the triton channel, we calculate the (exact) linear setup component as a convenient choice to study $3 \mathrm{NF}$. While we can access only a part of $3 \mathrm{NF}$ from it, 
we plan to extend the calculation to more general geometries step by step, toward the complete determination of the full $3 \mathrm{NF}$.

We consider the identification of genuine 3NF. It is a nontrivial work: Although both of parity-even and parity-odd $2 \mathrm{NF}$ are required to subtract $2 \mathrm{NF}$ part in Eq. (10), parity-odd $2 \mathrm{NF}$ have not been obtained yet in lattice QCD. (See, however, our recent progress [35.) In order to resolve this issue, we consider the following channel,

$$
\psi_{S} \equiv \frac{1}{\sqrt{6}}\left[-p_{\uparrow} n_{\uparrow} n_{\downarrow}+p_{\uparrow} n_{\downarrow} n_{\uparrow}-n_{\uparrow} n_{\downarrow} p_{\uparrow}+n_{\downarrow} n_{\uparrow} p_{\uparrow}+n_{\uparrow} p_{\uparrow} n_{\downarrow}-n_{\downarrow} p_{\uparrow} n_{\uparrow}\right],
$$

which is anti-symmetric in spin/isospin spaces for any $2 \mathrm{~N}$-pair. Combined with the Pauli-principle, it is automatically guaranteed that any $2 \mathrm{~N}$-pair couples with even parity only. Therefore, we can extract $3 \mathrm{NF}$ unambiguously using only parity-even $2 \mathrm{NF}$. Note that no assumption on the choice of 3D-configuration of $\mathbf{r}, \boldsymbol{\rho}$ is imposed in this argument, and we thus can take advantage of this feature for future $3 \mathrm{NF}$ calculations with various setup of $3 \mathrm{D}$-geometries.

\section{Lattice QCD setup and Numerical results}

We employ $N_{f}=2$ dynamical configurations with mean field improved clover fermion and RG-improved gauge action generated by CP-PACS Collaboration [41]. We use 598 configurations at $\beta=1.95$ and the lattice spacing of $a^{-1}=1.269(14) \mathrm{GeV}$, and the lattice size of $V=L^{3} \times T=16^{3} \times 32$ corresponds to $(2.5 \mathrm{fm})^{3}$ box in physical spacial size. For $u, d$ quark masses, we take the hopping parameter at the unitary point as $\kappa_{u d}=0.13750$, which corresponds to $m_{\pi}=1.13 \mathrm{GeV}, m_{N}=2.15 \mathrm{GeV}$ and $m_{\Delta}=2.31 \mathrm{GeV}$. Lattice simulations are performed at eleven physical points of the distance $r_{2}$. We use the wall quark source with Coulomb gauge fixing. In order to enhance the statistics, we perform the measurement on 32 wall sources using different time slices, and the forward and backward propagations are averaged. The results from both of total angular momentum $J_{z}= \pm 1 / 2$ are averaged as well. For the sink time, we calculate for a wide range of $5 \leq\left(t-t_{0}\right) / a \leq 10$. We evaluate Eq. (10) at $t$ being integer or half-integer, and we adopt the symmetric difference on the lattice for the time derivative. In the case of $t$ of integer, $\psi_{3 N}(\boldsymbol{r}, \boldsymbol{\rho}, t)$ is obtained directly on the lattice, while $\frac{\partial}{\partial t} \psi_{3 N}(\boldsymbol{r}, \boldsymbol{\rho}, t)$ is obtained from $\psi_{3 N}(\boldsymbol{r}, \boldsymbol{\rho}, t \pm 1)$. In the case of $t$ of half-integer, both of $\psi_{3 N}(\boldsymbol{r}, \boldsymbol{\rho}, t)$ and $\frac{\partial}{\partial t} \psi_{3 N}(\boldsymbol{r}, \boldsymbol{\rho}, t)$ are evaluated from $\psi_{3 N}(\boldsymbol{r}, \boldsymbol{\rho}, t \pm 1 / 2)$.

In Fig. 1] we plot the radial part of each wave function of $\psi_{S}=\left(-\psi_{1_{S_{0}}}+\psi_{3_{S_{1}}}\right) / \sqrt{2}$, $\psi_{M} \equiv\left(\psi_{{ }_{1} S_{0}}+\psi_{3 S_{1}}\right) / \sqrt{2}$ and $\psi_{3 D_{1}}$ obtained at $\left(t-t_{0}\right) / a=8$. Here, we normalize the wave functions by the central value of $\psi_{S}\left(r_{2}=0\right)$. What is noteworthy is that the wave functions are obtained with good precision, which is quite nontrivial for the $3 \mathrm{~N}$ system. We observe that $\psi_{S}$ overwhelms other wave functions. This indicates higher partial wave components are strongly suppressed, and the effect of the next leading order in the derivative expansion, spin-orbit forces, is suppressed in this lattice setup.

We determine $3 \mathrm{NF}$ by subtracting $2 \mathrm{NF}$ from total potentials in the $3 \mathrm{~N}$ system. Since we have only one channel (Eq. (11)) free from parity-odd $2 \mathrm{NF}$, we can determine one type of $3 \mathrm{NF}$. In this work, $3 \mathrm{NF}$ are effectively represented in a scalar-isoscalar functional form, which is often employed for phenomenological short-range 3NF 13 .

In Fig. 2, we plot the preliminary results for the effective scalar-isoscalar $3 \mathrm{NF}$ at $\left(t-t_{0}\right) / a=7.5,8.0,8.5$. The results from different sink times are consistent with each other within statistical fluctuations. This indicates that contaminations from excited states above inelastic threshold and/or the higher order terms in the velocity expansion 


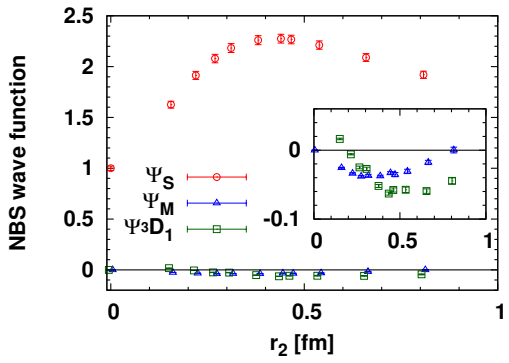

Fig. $13 \mathrm{~N}$ wave functions in the triton channel at $\left(t-t_{0}\right) / a=8$. Circle (red), triangle (blue), square (green) points denote $\psi_{S}, \psi_{M}$, $\psi_{{ }^{3} D_{1}}$, respectively. $r_{2}$ is the distance between the center and edge in the linear setup.

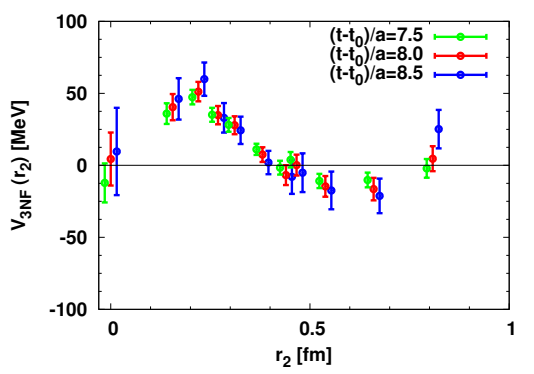

Fig. 2 The effective scalar-isoscalar $3 \mathrm{NF}$ in the triton channel with the linear setup. Green, red and blue points (with offset for visibility) are obtained at $\left(t-t_{0}\right) / a=7.5$, 8.0 and 8.5 , respectively.

of the potential are marginal. Fig. 2 shows that $3 \mathrm{NF}$ are small at the long distance region of $r_{2}$. This is in accordance with the suppression of $2 \pi \mathrm{E}-3 \mathrm{NF}$ by the heavy pion. At the short distance region, however, an indication of repulsive $3 \mathrm{NF}$ is observed. We recall that a repulsive short-range $3 \mathrm{NF}$ is phenomenologically required to explain the properties of high density matter. Since multi-meson exchanges are strongly suppressed by the large quark mass, the origin of this short-range $3 \mathrm{NF}$ may be attributed to the quark and gluon dynamics directly. In fact, we recall that the short-range repulsive (or attractive) cores in the generalized two-baryon potentials are systematically calculated in lattice QCD in the flavor SU(3) limit, and the results are found to be well explained from the viewpoint of the Pauli exclusion principle in the quark level [28,34]. In this context, it is intuitive to expect that the $3 \mathrm{~N}$ system is subject to extra Pauli repulsion effect, which could be an origin of the observed short-range repulsive 3NF. Further investigation along this line is certainly an interesting subject in future. It is also of interest that the analyses with operator product expansion [42] show that $3 \mathrm{NF}$ has a repulsive core at short distance.

Evaluation of systematic errors are in progress. In particular, the effect of the discretization error is important to be investigated, since the nontrivial results are obtained at short distance. For this purpose, an explicit lattice simulation with a finer lattice is currently underway. Quark mass dependence of $3 \mathrm{NF}$ is certainly an important issue as well, since the lattice simulations are carried out only at single large quark mass. In the case of $2 \mathrm{NF}$, short-range cores have the enhanced strength and broaden range by decreasing the quark mass $\underline{26}$. We, therefore, would expect a significant quark mass dependence exist in short-range $3 \mathrm{NF}$ as well. In addition, long-range $2 \pi \mathrm{E}-3 \mathrm{NF}$ will emerge at lighter quark masses, in particular, at the physical point. Quantitative investigation by lattice simulations with lighter quark masses are currently underway.

\section{Conclusions and Outlook}

We have explored three-nucleon forces (3NF) in lattice QCD, utilizing the imaginarytime Nambu-Bethe-Salpeter wave function. The enormous computational cost is drastically reduced by the newly-developed "unified contraction algorithm." Using $N_{f}=2$ dynamical clover fermion configurations at $a=0.156 \mathrm{fm}, V=16^{3} \times 32$ and $m_{\pi}=1.13$ 
$\mathrm{GeV}$, we have studied 3NF in which three nucleons are aligned linearly with an equal spacing. Repulsive 3NF have been found at short distance in the triton channel.

Currently, nuclear forces in lattice QCD are determined at rather heavy quark masses, which is considered to be a largest source of systematic errors. However, thanks to the significant theoretical and hardware development, it becomes possible to perform lattice simulations at the physical quark mass point. Generation of physical point gauge configurations with large volume has started, and nuclear forces will be subsequently studied 45. While there remain various challenges, it is becoming within reach to determine realistic nuclear forces including few-baryon forces from first-principles lattice simulations, which will play an ultimate role in nuclear physics and astrophysics.

Acknowledgements We thank authors and maintainers of CPS ++43 . We also thank CPPACS Collaboration and ILDG/JLDG 44] for providing gauge configurations. The numerical simulations have been performed on Blue Gene/L, Blue Gene/Q and SR16000 at KEK, SR16000 at YITP in Kyoto University, T2K at University of Tsukuba and T2K and FX10 at Tokyo University. This research is supported in part by MEXT Grant-in-Aid for Young Scientists (B) (24740146), Scientific Research on Innovative Areas (No.2004: 20105001, 20105003), the Large Scale Simulation Program of KEK, the collaborative interdisciplinary program at T2K-Tsukuba, and SPIRE (Strategic Program for Innovative REsearch).

\section{References}

1. S. C. Pieper, "Quantum Monte Carlo calculations of light nuclei," Riv. Nuovo Cim. 31, 709 (2008) arXiv:0711.1500 [nucl-th]].

2. T. Otsuka, T. Suzuki, J. D. Holt, A. Schwenk and Y. Akaishi, "Three-body forces and the limit of oxygen isotopes," Phys. Rev. Lett. 105, 032501 (2010) arXiv:0908.2607 [nucl-th]].

3. A. Akmal, V. R. Pandharipande and D. G. Ravenhall, "The Equation of state of nucleon matter and neutron star structure," Phys. Rev. C58, 1804 (1998) nucl-th/9804027.

4. K. Sekiguchi et al., "Three Nucleon Force Effects in Intermediate Energy Deuteron Analyzing Powers for dp Elastic Scattering," Phys. Rev. C 83, 061001 (2011) arXiv:1106.0180 [nucl-ex]].

5. K. Sekiguchi, "Experimental Approach to Three Nucleon Forces via Few Nucleon Systems," in these proceedings.

6. P. Demorest et al., "Shapiro Delay Measurement of A Two Solar Mass Neutron Star," Nature 467, 1081 (2010) arXiv:1010.5788 [astro-ph.HE]].

7. S. Nishizaki, T. Takatsuka and Y. Yamamoto, "Hyperon-mixed neutron star matter and neutron stars," Prog. Theor. Phys. 108, 703 (2002).

8. T. Takatsuka, S. Nishizaki and R. Tamagaki, "Three-body force as an 'extra repulsion' suggested from hyperon-mixed neutron stars," Prog. Theor. Phys. Suppl. 174, 80 (2008).

9. J. Fujita and H. Miyazawa, "Pion Theory of Three-Body Forces," Prog. Theor. Phys. 17, 360 (1957).

10. S. A. Coon and H. K. Han, "Reworking the Tucson-Melbourne three nucleon potential," Few Body Syst. 30, 131 (2001) arXiv:nucl-th/0101003.

11. A. Nogga, D. Huber, H. Kamada and W. Gloeckle, "Benchmark calculations for the triton binding energy for modern N N forces and the pi pi exchange three nucleon force," Phys. Lett. B 409, 19 (1997) nucl-th/9704001.

12. R. Machleidt, K. Holinde and C. Elster, "The Bonn Meson Exchange Model for the Nucleon Nucleon Interaction," Phys. Rept. 149, 1 (1987).

13. S. C. Pieper, V. R. Pandharipande, R. B. Wiringa and J. Carlson, "Realistic models of pion exchange three nucleon interactions," Phys. Rev. C 64, 014001 (2001) arXiv:nucl-th/0102004.

14. R. Machleidt and D. R. Entem, "Chiral effective field theory and nuclear forces," Phys. Rept. 503, 1 (2011) arXiv:1105.2919 [nucl-th]].

15. R. Machleidt, Q. MacPherson, E. Marji, R. Winzer, C. .Zeoli and D. R. Entem, "Recent Progress in the Theory of Nuclear Forces," arXiv:1210.0992 [nucl-th], in these proceedings.

16. E. Epelbaum et al., "Three nucleon forces from chiral effective field theory," Phys. Rev.

C 66, 064001 (2002) nucl-th/0208023. 
17. R. Skibinski, J. Golak, K. Topolnicki, H. Witala, E. Epelbaum, W. Glockle, H. Krebs and A. Nogga et al., "The triton with long-range chiral N3LO three nucleon forces," Phys. Rev. C 84, 054005 (2011) arXiv:1107.5163 [nucl-th]].

18. T. Doi [HAL QCD Collaboration], "The study of the Three Nucleon Force in full QCD Lattice calculations," PoS LATTICE2010, 136 (2010) arXiv:1011.0657 [hep-lat]].

19. T. Doi et al. [HAL QCD Collaboration], "Exploring Three-Nucleon Forces in Lattice QCD," Prog. Theor. Phys. 127, 723 (2012) arXiv:1106.2276 [hep-lat]].

20. T. Yamazaki, Y. Kuramashi and A. Ukawa, [PACS-CS Collaboration], "Helium Nuclei in Quenched Lattice QCD," Phys. Rev. D81, 111504 (2010) arXiv:0912.1383 [hep-lat]].

21. T. Yamazaki, K. -I. Ishikawa, Y. Kuramashi and A. Ukawa, "Helium nuclei, deuteron and dineutron in 2+1 flavor lattice QCD," Phys. Rev. D 86, 074514 (2012) arXiv:1207.4277 [hep-lat]].

22. S. R. Beane et al., "High Statistics Analysis using Anisotropic Clover Lattices. II. ThreeBaryon Systems," Phys. Rev. D 80, 074501 (2009) arXiv:0905.0466 [hep-lat]].

23. S. R. Beane et al., "Light Nuclei and Hypernuclei from Quantum Chromodynamics in the Limit of SU(3) Flavor Symmetry," arXiv:1206.5219 [hep-lat].

24. N. Ishii, S. Aoki and T. Hatsuda, "The Nuclear Force from Lattice QCD," Phys. Rev. Lett. 99, 022001 (2007) nucl-th/0611096.

25. S. Aoki, T. Hatsuda and N. Ishii, "Nuclear Force from Monte Carlo Simulations of Lattice Quantum Chromodynamics," Comput. Sci. Dis. 1, 015009 (2008) arXiv:0805.2462 [hep-ph]].

26. S. Aoki, T. Hatsuda and N. Ishii, "Theoretical Foundation of the Nuclear Force in QCD and its applications to Central and Tensor Forces in Quenched Lattice QCD Simulations," Prog. Theor. Phys. 123, 89 (2010) arXiv:0909.5585 [hep-lat]].

27. H. Nemura, N. Ishii, S. Aoki and T. Hatsuda, "Hyperon-nucleon force from lattice QCD," Phys. Lett. B673, 136 (2009) arXiv:0806.1094 [nucl-th]].

28. T. Inoue et al. [HAL QCD Collaboration], "Baryon-Baryon Interactions in the Flavor SU(3) Limit from Full QCD Simulations on the Lattice," Prog. Theor. Phys. 124, 591 (2010) arXiv:1007.3559 [hep-lat]].

29. K. Sasaki [HAL QCD Collaboration], "Lattice QCD study of baryon-baryon interactions in the $(\mathrm{S}, \mathrm{I})=(-2,0)$ system using the coupled-channel formalism," PoS LATTICE2010, 157 (2010) arXiv:1012.5685 [hep-lat]].

30. T. Inoue et al. [HAL QCD Collaboration], "Bound H-dibaryon in Flavor SU(3) Limit of Lattice QCD," Phys. Rev. Lett. 106, 162002 (2011) arXiv:1012.5928 [hep-lat]].

31. S. Aoki et al. [HAL QCD Collaboration], "Extraction of Hadron Interactions above Inelastic Threshold in Lattice QCD," Proc. Jpn. Acad. Ser. B 87, 509 (2011) arXiv:1106.2281 [hep-lat]].

32. Y. Ikeda [for HAL QCD Collaboration], "S-wave meson-baryon potentials with strangeness from Lattice QCD," PoS LATTICE 2011, 159 (2011) arXiv:1111.2663 [hep-lat]].

33. K. Murano, N. Ishii, S. Aoki and T. Hatsuda, "Nucleon-Nucleon Potential and its Nonlocality in Lattice QCD," Prog. Theor. Phys. 125, 1225 (2011) arXiv:1103.0619 [hep-lat]].

34. T. Inoue et al. [HAL QCD Collaboration], "Two-Baryon Potentials and H-Dibaryon from 3-flavor Lattice QCD Simulations," Nucl. Phys. A 881, 28 (2012) arXiv:1112.5926 [hep-lat]].

35. K. Murano [HALQCD Collaboration], "Nuclear forces in the parity odd sector and the LS forces," PoS LATTICE 2011, 319 (2011) arXiv:1112.2051 [hep-lat]].

36. N. Ishii et al. [HAL QCD Collaboration], "Hadron-Hadron Interactions from Imaginarytime Nambu-Bethe-Salpeter Wave Function on the Lattice," Phys. Lett. B 712, 437 (2012) arXiv:1203.3642 [hep-lat]].

37. S. Aoki [HAL QCD Collaboration], "Hadron interactions in lattice QCD," Prog. Part. Nucl. Phys. 66, 687 (2011) arXiv:1107.1284 [hep-lat]].

38. S. Aoki et al. [HAL QCD Collaboration], "Lattice QCD approach to Nuclear Physics," Prog. Theor. Exp. Phys. 2012, $01 \mathrm{~A} 105$ (2012) arXiv:1206.5088 [hep-lat]].

39. T. Doi and M. G. Endres, "Unified contraction algorithm for multi-baryon correlators on the lattice," Comput. Phys. Commun. 184, 117 (2013) arXiv:1205.0585 [hep-lat]].

40. S. Aoki et al. [HAL QCD Collaboration], in preparation.

41. A. Ali Khan et al. [CP-PACS Collaboration], "Light hadron spectroscopy with two flavors of dynamical quarks on the lattice," Phys. Rev. D 65, 054505 (2002) [E: D 67, 059901 (2003)].

42. S. Aoki, J. Balog and P. Weisz, "Short distance repulsion in 3 nucleon forces from perturbative QCD," New J. Phys. 14, 043046 (2012) arXiv:1112.2053 [hep-lat]]; ibid., "Toward an understanding of short distance repulsions among baryons in QCD - NBS wave functions and operator product expansion -," arXiv:1208.1530 [hep-lat]. 
43. Columbia Physics System (CPS), "http://qcdoc.phys.columbia.edu/cps.html"

44. "http://www.lqcd.org/ildg", "http://www.jldg.org"

45. "http://www.jicfus.jp/field5/en/" 